\title{
Misión y desempeño docente
}

\section{Abreu Corona, Arelis Milagros*}

\section{Resumen}

Este estudio estuvo orientado a determinar la relación entre la Dirección de la Misión Organizacional y el Desempeño Docente de la Escuela Técnica. Hno. Ildefonso Gutiérrez, en Maracaibo, Venezuela. El diseño aplicado es de tipo transversal y la técnica empleada para la recolección de datos fue un instrumento mixto (prueba de aprovechamiento y escala de frecuencia tipo Likert) para evaluar la Dirección de la Misión de la organización, la cual fue aplicada a toda la población docente de la institución. Asimismo se aplicó un cuestionario con una escala de acuerdos a una muestra de 20 alumnos para evaluar el desempeño docente, tomando en cuenta características personales, rasgos pedagógicos y funciones. El análisis descriptivo-correlacional arrojó como resultado un nivel óptimo reflejado con un $76,79 \%$ en: (conocimiento de la misión), $82.58 \%$ (valores corporativos) y $90.91 \%$ (desempeño docente). De igual forma se aplicó la correlación entre las variables obteniéndose un coeficiente $r=0.43$ para un nivel de $p<0.001$. Se concluyó que existe una relación muy significativa entre la misión organizacional y el desempeño de los docentes. Este hallazgo permite inferir que las organizaciones educativas deben tomar la misión como elemento de dirección por lo que los gerentes deben establecer criterios de desempeño para evaluar a los docentes con parámetros congruentes con la misión de la institución que ellos administren.

Palabras clave: Misión educativa, rendimiento docente, evaluación institucional, organizaciones educativas, Venezuela. 


\section{Teaching Mission and Performance}

\section{Abstract}

This study is oriented toward determinig the relationaship between the Organizational Mission and Teaching Performance at Vocational School Hno. Idelfonso Gutierrez. The design employed in data collection was the application of mixed instruments, (a test of achievement and a Likert style scale) in order to evaluate the orientation of the organizational mission. They were applied to all teachers in the institution. At the same time, a questionaire was applied to a sample of 20 students to evaluate teacher performance, taking into account personal characteristics, pedagogical characteristics and pedagogical functions. The descriptive-correlational analysis resulted in optimum levels reflected in a value of $76.79 \%$ (knowledge of mission), $82.58 \%$ (corporative values) and $90.91 \%$ (teacher performance). In the same manner the correlation between the variables gave a r-coefficient value of 0.43 at a $p<0.001$ level. The conclusion is that there is a very significant relation between organizational mission and teacher performance. This finding allows us to infer that educational organizations should use mission as a directive element since managers must establish performance criteria to evaluate teachers on the basis of congruency with the institutional mission of the institution they administer.

Key words: Educational mission, teacher performance, institutional evaluation educational organizations.

\section{Introducción}

La década de los 90 ha sido escenario de grandes acontecimientos en el campo de los estudios gerenciales y en desarrollo organizacional. Los avances tecnológicos, la competitividad y las transformaciones sociales hacen más complejo el mundo actual. Esto ha hecho que las organizaciones se enfrenten a situaciones y retos cada vez más difíciles, donde el conocimiento técnico se encuentra en abundancia, y sigue creciendo con el paso de los años.

Por ello, los líderes empresariales hacen frente hoy más que en cualquier momento del pasado, a la necesidad de definir con claridad sus organizaciones o de redefinirlas. Existe una creciente crisis de significado y un constante reclamo de visión y misión en el sentido de dirección y orientación, ya que la mayoría de las organizaciones no saben quiénes son, cuál es la lógica de su existencia, ni mucho menos se preocupan en direccionar a través de sus valores.

Esto ha llevado a que teóricos e investigadores acepten y reconozcan el importante papel que desempeña la declaración de la visión y misión en la vida de la organización, pues a través de ella se expresan los propósitos, valores y 
prioridades con los cuales se identifican, estableciéndose asi un marco de referencia que va a indicar cuál es el modo de pensar y actuar esperado en los miembros de esa organización.

De alli que autores como Davis (1994), Quigley (1996), Albrecht (1996), y Serna (1997) coincidan en formular que la misión es una tarea gerencial de mucha importancia, ya que por medio de ella cada empresa se distingue de otra a partir de su contenido y dimensión. En ella se señalan las prioridades, la razón de su existencia, se identifican los mercados a los cuáles se dirige, los clientes a quienes desean servir y los productos o servicios que quiere ofrecer.

Es importante destacar que los autores antes señalados consideran que el conocimiento de la misión crea compromiso, asegura consistencia y claridad de los propósitos, así como también induce y direcciona a crear comportamientos cónsonos con los valores y filosofía de la institución. Una organización que no tiene desarrollada una formulación explícita de la misión induce que la labor de los empleados se desvanezca con gran facilidad.

Las organizaciones que logran obtener un alto nivel de productividad es porque consiguen niveles superiores de desempeño y rendimiento de sus empleados (Dalziel, et al, 1996:9). Este rendimiento se logra si los empleados se sienten comprometidos y motivados a cumplir a cabalidad con las funciones que le han sido encomendadas en su lugar de trabajo. De alli, que el desempeño y la satisfacción son factores que están estrechamente vinculados con la misión, ya que la internalización de la misma facilita la realización de tareas y de objetivos de la organización.

Lo anteriormente expuesto induce a señalar la importancia de la dirección de la misión y el desempeño para el logro de la efectividad en las organizaciones. Este planteamiento es válido para cualquier tipo de organización; por lo tanto, las organizaciones educativas no escapan a esta necesidad.

Para lograr consolidar la imagen de la institución educativa tanto a nivel interno como externo se requiere que sus miembros se identifiquen con su filosofía y valores corporativos, elementos importantes que van a ser reflejados en la misión y transmitidos a través de su comportamiento y desempeño docente. En una institución educativa donde se desconozca la declaración de la misión y los valores organizacionales, dificilmente sus miembros sabrán el por qué deben cumplir sus funciones.

Para lograr que las organizaciones educativas sean exitosas se requiere que se formule una visión y misión clara, que proyecte una idea impuisora efectiva para movilizar a las personas hacia un mismo fin, que sirva de punto de referencia para tomar la dirección correcta hacia él, permitiendo de esta manera que sus miembros desempeñen sus labores no sólo para vivir, sino porque persiguen un propósito superior, el cual debe ser reflejado en el valor de la persona que se está formando. Por ello la discusión sobre el efecto que el conocimiento de la misión tenga sobre la dirección del desempeño laboral es un punto álgido que debe ser abordado en cualquier organización. 
Es por ello que los gerentes educativos deben establecer criterios de desempeño a sus docentes, para poder evaluarlos en base a parámetros congruentes con la misión de la institución.

En esta investigación se planteó como objetivo general determinar la relación que existe entre la dirección de la misión organizacional y el desempeño docente de la Escuela Técnica Hno. Ildefonso Gutiérrez. De igual forma se plantearon los siguientes objetivos especificos: Identificar el nivel de conocimiento de la misión de la organización por parte de los docentes, identificar los valores corporativos en los docentes y determinar el nivel de desempeño de los mismos.

El tipo de investigación es correlacional, ya que tiene como propósito medir la relación entre las dos variables (Misión y Desempeño Docente). Se hizo un estudio tipo censo, para la cual se tomó toda la población objeto de estudio (42 docentes). El diseño que se utilizó fue de campo de tipo transeccional correlacional.

La formulación teórica que se expone a continuación contiene las dimensiones fundamentales que sustentan las variables misión y desempeño docente. En cuanto a los valores, se hace necesario hacer énfasis en este elemento de la misión, pues es considerado factor fundamental en la dirección y orientación de los miembros de la organización.

\section{Reflexiones teóricas sobre la Misión Organizacional: su aplicación a instituciones educativas}

La visión constituye la declaración de los valores y propósitos de una organi- zación, por lo que debe ser amplia y suficiente que motive y promueva la pertenencia de todos los miembros de la organización.

La visión de acuerdo a Quigley (1996:80) constituye la declaración más fundamental de los valores, aspiraciones y metas de una corporación. De acuerdo a Serna (1997:21), "es un conjunto de ideas generales que proveen el marco de referencia de lo que una empresa es y quiere ser en el futuro", ahora bien, todas las organizaciones tienen básicamente las mismas razones para justificar su existencia: sobrevivir, crecer, proveer un servicio o producto. Sin embargo, las organizaciones tienen que ir más allá de estas razones básicas para diferenciarse en el campo de trabajo. Esta diferenciación debe expresarse a través de la misión y propósitos de la organización, por lo que David (1994:7) expresa que la misión de una organización es la formulación de un propósito duradero que distingue a una empresa de otra, identifica el alcance de la operaciones del producto y del mercado. Asimismo revela las necesidades principales del cliente que la firma se propone satisfacer.

Esta formulación del propósito a la que se refiere el autor antes mencionado, es llamada también declaración del credo, de filosofia, de principios empresariales, cualquiera que sea el término que se utilice revela el concepto de una organización, su principal producto o servicio, por lo que debe ser clara y significativa, ya que va a describir las características más relevantes de la organización.

Por otra parte Albrecht (1996:80) refiere que la misión organizacional es un enunciado simple y preciso del modo como 
la empresa debe hacer negocios, por lo que debe definir a sus clientes, la premisa de valor que le ofrece, y cualquier medio especial que se utilice para la creación de un valor que cree interés en el cliente.

De igual forma Quincley (1996:30) ratifica que la misión es el elemento de la visión corporativa que debe responder en la organización ¿Qué somos hoy?, ¿Qué aspiramos ser?, ¿Cuál es nuestra principal capacidad? Estas preguntas van a permitir una comprensión clara de lo que hay que cambiar y hacia donde ir.

Asimismo Serna (1997:26), afirma que la misión es "la formulación de los propósitos de una organización que la distingue de otros negocios, de acuerdo a sus principios, valores, cubrimiento de sus operaciones, sus productos, mercados y el talento humano que soporta el logro de estos propósitos".

Serna coincide con la posición de los autores antes mencionados en cuanto a la misión o propósito de la organiza- ción, sin embargo manifiesta que ésta debe describir de manera amplia la naturaleza del negocio a la cual se dedica la organización por lo que debe responder en términos generales a una serie de preguntas (Ver gráfico 1).

A objeto de precisar cada uno de los elementos de la misión organizacional siguiendo a Serna y extrapolándolo a las organizaciones sin fines de lucro se explicará lo que se entiende por cada una de ellas.

¿Cuál es el negocio? Esta pregunta es el punto esencial en la definición de la misión, ya que a través de ella se da a conocer el tipo de empresa y las características que ésta posee. Para organizaciones sin fines de lucro este elemento debe responder a la pregunta: ¿A qué función sirve la organización?, ya que la pregunta en general en el ámbito organizacional debe responder en términos generales a la función que intenta cumplir.

\section{Gráfico 1}

Elementos de la Misión Organizacional

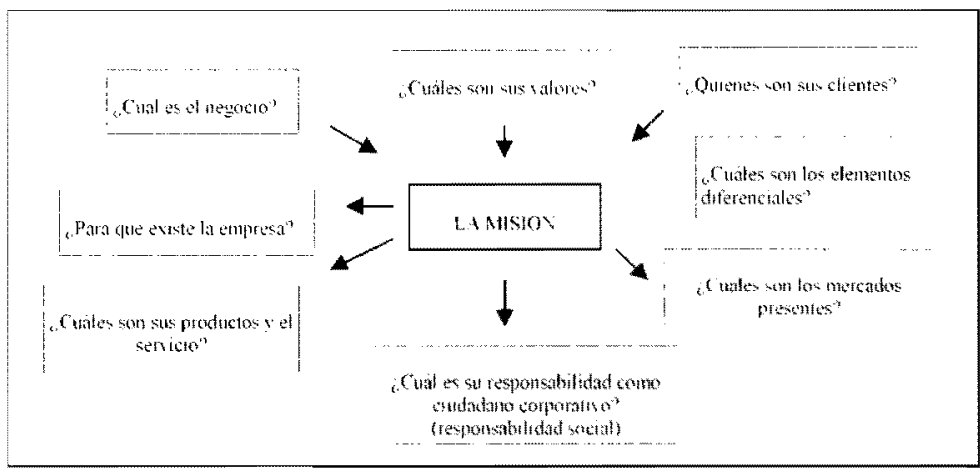

Fuente: Serna Gómez (1996). 
¿Para qué existe la empresa? ¿Cuál es el propósito básico? Explica la existencia de la organización en cuanto a sus propósitos, contribución y responsabilidad social. Es decir tanto para organizaciones con o sin fines de lucro esta pregunta invita a analizar los motivos de su creación, tales como contribución al de. sarrollo del país, logro de visión empresarial, entre otras.

¿Quiénes son nuestros clientes? Se debe expresar de manera clara cuáles son los clientes, ya que las organizaciones dependiendo de sus productos o servicios tienen diferentes tipos de clientes a los cuales es indispensable satisfacer en sus necesidades y expectativas. Se entiende por cliente el ente principal al que se intenta servir. Extrapolando esta argumentación a las organizaciones sin fines de lucro, la pregunta debe responder a quién van dirigidos los productos o servicios que ofrece.

¿Cuáles son los elementos diferenciales? Este elemento se refiere a las aptitudes distintivas que diferencian a una organización de sus competidores y que se mide a través de los productos y servicios que se ofrecen a un cliente satisfecho. De igual forma, en las organizaciones sin fines de lucro es necesario puntualizar en esta diferenciación, ya que por medio de ella se van a establecer las fortalezas y ventajas competitivas, claves con que la institución va a contar como recurso básico para ofrecer un servicio que logre alto grado de satisfacción en el cliente.

¿Cuáles son los servicios presentes y futuros? En la misión deben expresarse claramente los productos o servicios que clientes, usuarios o consumido- res van a recibir de la empresa dependiendo del tipo y características de la organización. Para el caso de las organizaciones educativas, se deben expresar claramente los servicios que se ofrecen para satisfacer las necesidades que demanda el entorno, el cual cambia sus características básicas dependiendo de las condicionantes socioeconómicas educativas prevalecientes.

¿Cuáles son los valores organizacionales? Esta pregunta es considerada relevante, pues en la misión debe hacerse referencia a los principios y valores corporativos, con el fin de incorporarlos al quehacer diario de la organización, y hacerlo parte de la definición del negocio o razón de ser para las organizaciones lucrativas y no lucrativas.

¿Cuáles son los mercados presentes? Este elemento se refiere al mercado al cual va dirigido el producto o servicio, tomando en cuenta su situación geográfica (regional, nacional e internacional). En el caso de las organizaciones educativas este factor responde al ámbito de competencia, es decir, el entorno o espacio geográfico que se cubre con el servicio que se ofrece y de acuerdo con las necesidades específicas que surgen de la población estudiantil inmersa en ese espacio.

¿Cuál es la responsabilidad corporativa y social? Esta pregunta debe dar respuesta a la responsabilidad y compromiso que tiene la organización ante los accionistas, colaboradores, clientes, la competencia, el medio ambiente y la sociedad en general.

Esta teoría puede ser aplicada a todo tipo de organización, ya que cada una de ellas es única y como tal posee su propia identidad, principios, valores y ob- 
jetivos, los cuales son difundidos a través de su misión, por lo que se pueden extrapolar los términos de la teoría presentada por el autor Serna (1997: 22) a las instituciones educativas, con la finalidad de describir la naturaleza de la organización y poder de esta manera obtener una misión clara, que pueda ser captada por sus miembros.

Ante la misión de las instituciones educativas se respondería a las preguntas: ¿A qué función sirve?, ¿A quién sirve?, ¿Cuál es el propósito de la institución?, ¿Cuáles son sus servicios?, ¿Cuál es el ámbito de competencia?, ¿Cuáles son sus valores?, ¿Cuáles son sus fortalezas?, ¿Cuál es la responsabilidad social?. (Ver gráfico 2). Estas preguntas constituyen la clave del éxito en la definición de la misión ya que dan respuestas al tipo de institución educativa, sus características, objetivos, razón de existencia, valores y servicios que ella ofrece y que la hacen diferente a las demás.

De allí que se puede decir que la misión organizacional es la formulación de los propósitos, función, fortalezas, ámbito de competencia, valores y servicios que la institución educativa ofrece a los alumnos y que la distinguen de otras organizaciones, de acuerdo con su filosofía, gestión, administración, y recurso humano que hacen posible el logro de estos propósitos.

\section{Sobre los valores corporativos y misión}

Los valores nos dicen lo que es ético, bueno, válido, competitivo, adecuado, hermoso o deseable, y se van generando y reforzando a lo largo de la vida. Según García y Dolan (1997:62) la palabra valor tiene en castellano y en otras lenguas románicas 3 diferentes acepciones que resultan complementarias para entender la importancia para dirigir mediante valores. Estas acepciones hacen referencia a la:

Dimensión ético-estratégica: las elecciones preferenciales. Los valores constituyen el núcleo de la libertad humana en cuanto a que éstos constituyen elecciones deliberadas $\circ$ preferencias estratégicas (a mediano-largo plazo) que las personas eligen preferentemente dentro de un conjunto dado de valores y lo asumen como normas de conducta automática.

Dimensión económica: La valía. Son criterios utilizados para evaluar las personas, objeto, ideas, actos, sentimientos o hechos en cuanto a su relativo mérito, adecuación, escasez, precio o interés.

Dimensión Psicológica: La valentía. García y Dolan (1997: 65) refieren que el diccionario define a la palabra valor como: "cualidad moral que mueve a cometer resueltamente grandes empresas y afrontar sin miedo los peligros". Esta última definición incluye, de hecho, los tres criterios de la definición valor lo cual es de vital importancia, ya que puede afirmarse que la orientación del esfuerzo mediante valores aumenta la valentía de los individuos y grupos.

Quigley (1996:16) define a los valores corporativos como "las reglas o pautas mediante las cuales una corporación exhorta a sus miembros a tener comportamientos consistentes con su sentido de orden, seguridad, desarrollo".

Garcia y Dolan (1997:35) hacen referencia que toda empresa ha de tener 


\section{Gráfico 2}

Elementos de la Misión en las Organizaciones Educativas



Fuente: Elaboración propia.

definidos dos grandes grupos de valores o principios compartidos que orienten sus objetivos de acción cotidiana. 1) Los valores finales asociados con su visión (¿hacia dónde vamos) y su misión (razón de ser o (para qué?) y 2) Los valores instrumentales $u$ operativos asociados a la forma de pensar y hacer las cosas con que la organización pretende afrontar las demandas de su entorno e integrar sus tensiones internas para alcanzar su visión y misión.

Al hablar de valores es importante establecer la diferencia entre valores finales y valores de tipo instrumental. Los valores finales son esenciales para dar sentido y cohesionar el esfuerzo de hacia dónde va la empresa a largo plazo y hace referencia al tipo de negocio que se quiere llegar a ser, la dimensión a alcanzar y la diferenciación que se pretende asumir $y$ otros. De hecho, los valores integrados en la visión y misión definen las elecciones estratégicas esenciales de la empre- sa. Los valores instrumentales son modos de conductas adecuadas o necesarias para conseguir nuestras finalidades o valores existenciales.

Si tomamos en consideración que los valores son aprendizajes estratégicos relativamente estables de formas de actuar para conseguir un fin determinado, se puede decir que las organizaciones educativas deberían formular de manera clara los valores a través de su misión como estrategia fundamental para lograr la cohesión y motivación de sus miembros hacia las tareas y objetivos de la misma, ya que la falta de valores claramente formulados ocasiona desinterés, desmotivación y falta de compromiso con la misión.

Las empresas poseen una serie de valores relacionados con la razón de ser de la misma, estos valores se consideran como finales o existenciales y para poder alcanzarlos las organizaciones deben fomentar, motivar y dirigir aquellos valores instrumentales u operativos que de 
acuerdo con su criterio, los empleados deben poner en práctica en su quehacer diario para alcanzar aquellos valores implícitos en la misión y visión de la organización. Es la orientación estratégica del esfuerzo dirigido mediante los valores expuestos en la misión, lo que va a activar e inducir a sus miembros a obtener conductas valiosas y emprendedoras que logren que el trabajo sea una satisfacción plena, tanto para el individuo como para el grupo, comunidad y sociedad.

\section{Misión y Valores en la Escuela Técnica Hno. Ildefonso Gutiérrez}

La Escuela Técnica Hno. Ildefonso Gutiérrez, es una Asociación Civil sin fines de lucro, subvencionada por el Ministerio de Educación, ubicada en el Barrio los Estanques, en la ciudad de Maracaibo, Estado Zulia (Venezuela). Fue creada en 1966 por la Congregación Marista como respuesta de "Escuela Profesional". Su función es la educación integral de jóvenes de escasos recursos, útil para el trabajo productivo.

El propósito básico es formar técnicos medios en las áreas industriales y comerciales, con alto nivel de productividad y calidad, dispuestos a transformar la cultura del trabajo y desarrollo productivo del país.

El servicio que ofrece es una educación de calidad que busca la excelencia en los jóvenes de escasos recursos, que requieran de una titulación de técnico para trabajar y mantener sus familias y su desarrollo integral. Su responsabilidad social es formar jóvenes profesionales capaces de desempeñarse como buenos cristianos y honestos ciudadanos.

El ámbito en el cual la institución compite está referido a un espacio geográfico regional delimitado por una población de escasos recursos, con necesidades urgentes de formarse en una carrera corta para insertarse al mercado laboral.

Las fortalezas con las cuales cuenta la institución: alto nivel profesional, carisma del educador y ambiente de trabajo.

Sus valores finales: Formación de excelentes cristianos y honrados ciudadanos con alto nivel de calidad, valores cristianos, sentido de responsabilidad y cultura solidaria.

En la Unidad Educativa que se toma como objeto de estudio, el planteamiento de los valores corporativos se recoge en los principios y filosofía que se formulan para el Educador Marista de acuerdo con Escorihuela (1983: 82-120), expuestos por su fundador Marcelino Champagnat, con un sentido visionario, desde 1816 adelantado a su tiempo y quien utilizó su intuición para estructurar un sistema educativo direccionado porvalores que hoy es corroborado por las nuevas teorias organizacionales que introducen la dimensión humana dentro del pensamiento directivo y como práctica diaria. El impulso que da esta noción de dirección radica en la humanización del propósito básico de la organización, formar excelentes cristianos y honrados ciudadanos, hombres de bien que se articulen armónicamente en el sector productivo regional como técnicos medios en las áreas industriales y comerciales, con sentido de responsabilidad, preparados para enfrentar la vida y defender con eficacia la fe que profesan. Formados 
en una cultura solidaria, de amor a la patria, al trabajo y a sus semejantes. (Ver gráfico 3).

Según Escorihuela (1983: 83) los valores corporativos operativos con los que la institución logra conseguir su misión son:

- Devoción a María: valor esencial y espiritual de los maristas. Guía, compañera de camino en la misión del educador. Modelo predilecto de vida sencilla y de educación.

- Presencia del educador: Presencia que es cercanía, acompañamiento, entrega personal de tiempo y cordialidad, que se prolonga y se aproxima al alumno en actitud de aprendizaje, trabajo y orden.

- Vida de familia: valor esencial que se refleja en el afecto, servicio, acogida y hospitalidad.

- Sencillez: Valor humano que se refleja en el trato espontáneo, la alegría y actitud de servicio.

- Formación integral: Valor fundamental en la formación del individuo don- de se trata de unir en un solo acto la adquisición de la fe y del saber, de tal manera que las enseñanzas impartidas sirvan para prepararlo como persona que le ayude a servir a Dios y al prójimo en el ámbito donde se encuentre.

- Solidaridad: Valor esencial manifestado en el interés por la persona, expresada a través de la colaboración, cooperación y ayuda hacia los jóvenes, familias, comunidad.

Estos valores constituyen la esencia del quehacer diario y el estilo de vida de la institución, con la cual afronta de manera diferente la tarea educativa y se logra alcanzar los valores finales propuestos en la formulación de la misión organizacional.

\section{Reflexiones teóricas sobre desempeño docente}

Milkovichg (1994:96) manifiesta que "el desempeño del empleado es el grado en el cual éste cumple con los re-

\section{Gráfico 3}

\section{Valores finales de la misión organizacional Escuela Técnica} Hno. Ildefonso Gutiérrez

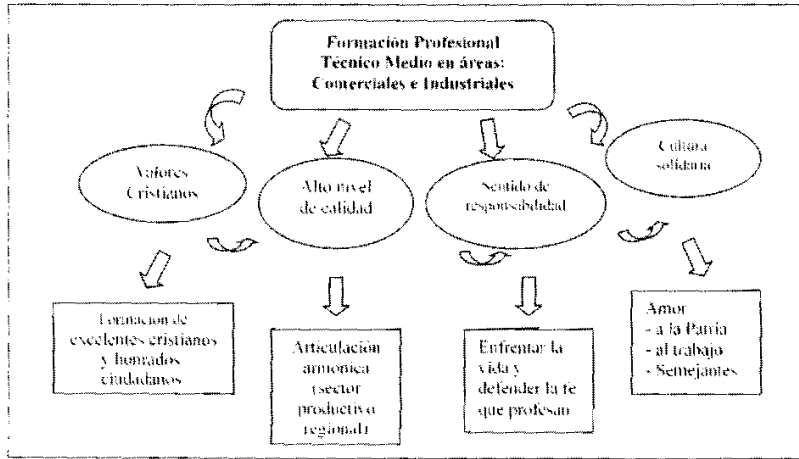

Fuente: Encuesta propia. 
quisitos de trabajo". En la medida que los empleados cumplan con efectividad los requisitos de su trabajo, éste redundará en beneficio para la empresa, logrando así mayor rendimiento y productividad. Para estos aspectos en las organizaciones educativas, se requiere que sus miembros se desempeñen de manera efectiva en su puesto de trabajo.

De acuerdo con el Reglamento de la Profesión Docente (1991:3) en su Art. 3 el desempeño docente "es el cumplimiento de aquellos deberes inherentes a la función que un docente debe llevar a cabo". Tomando en consideración a Milkovichg y al Reglamento antes mencionado se pudiese decir que el desempeño docente es el grado en el cual éste cumple con los requisitos y deberes inherentes a la función que como docente debe llevar a cabo.

Es importante destacar que la Ley Orgánica de Educación (1980:26) en su Art. 77 expresa 4 funciones fundamentales que debe cumplir el docente. Ellas son: enseñanza, orientación, planificación y evaluación. Por consiguiente, constituye un aporte valioso al desempeño del docente.

Al concebirse un proceso donde el educando interviene activamente en su aprendizaje, y donde se persigue el desarrollo integral de su personalidad en los aspectos físicos, intelectual, social, moral y emocional, el docente ya no podrá ser un simple transmisor de conocimiento, sino que deberá cumplir con funciones más relevantes que las que hasta ahora ha desempeñado, por lo que a raíz de la implantación de la Escuela Básica, el Ministerio de Educación propuso un perfil docente que se adaptara a las nuevas ne- cesidades imperantes. Tal es el caso de la función docente como facilitador del aprendizaje, orientador, investigador, promotor social y evaluador.

Como facilitador, se requiere que el docente propicie oportunidades y experiencias de aprendizaje, que estimulen la actividad de los educandos, a fin de lograr aprendizajes significativos para ellos.

Con respecto a la función de orientador, el docente proporcionará al educando atención como persona, tomará en cuenta sus características, necesidades $e$ intereses. De acuerdo con la función de investigador, el docente debe utilizar los resultados de las investigaciones como base para formular objetivos, reformular procedimientos, enriquecer metodologías y técnicas, así como estimular en los educandos el aprendizaje por vía del descubrimiento y la utilización de los procesos de investigación.

Como evaluador, el docente debe elaborar instrumentos prácticos que le permitan obtener información válida $y$ confiable del rendimiento del alumno. Así mismo, debe tomar en cuenta las caracteristicas biopsicosocial, motivaciones, dificultades en el aprendizaje del alumno de tal manera que pueda brindar una evaluación empleando un proceso integral, continuo, científico y flexible que le permita identificar, obtener, interpretar y suministrar información útil para la toma de decisiones en todas las etapas de la acción educativa.

Para efectos de esta investigación, se tomaron en consideración las funciones anteriormente descritas para mediry valorar en términos conductuales el desempeño docente como facilitador, orien- 
tador, evaluador e investigador, entre otros.

Por otra parte, existen otras acciones que forman parte del desempeño profesional del docente, se relacionan con aspectos administrativos como la puntualidad y la asistencia, pero también tienen que ver con rasgos de la personalidad y actitudes personales como es el caso de la responsabilidad y la participación en actividades conjuntas o cooperativas. De igual forma, el desempeño del docente va a depender en parte del conocimiento, habilidades, destrezas y la motivación que tenga éste dentro de la institución.

Robbins (1996: 702), manifiesta que uno de los factores que contribuye a mejorar el desempeño es el hecho que los valores del empleado, en este caso del docente, armonicen con los valores de la empresa. Para ello las organizaciones educativas deben definir e informar a los docentes acerca de los valores que aspiran, de tal manera que los docentes se sientan identificados con estos valores y satisfechos de su labor.

El evaluar a una persona tiene ciertas implicaciones como la percepción de conductas relacionadas con la ejecución. En este sentido, la ejecución laboral está conformada por actividades que son tangibles, observables y medibles de alguna manera y por otras que necesariamente hay que inferirlas.

En el caso de las organizaciones educativas, los administradores deben estimar o evaluar el desempeño del docente, centrándose en lo que éste llevó a cabo durante el año escolar, con la finalidad de verificar el alcance de los objetivos y metas propuestas por la organiza- ción. Los criterios de estimación deben estar de acuerdo a la conveniencia y propósitos de la institución para poder evaluar el rendimiento de sus docentes en función de los valores, caracteristicas, objetivos y metas propuestas por ella.

Tomando en consideración la teoría antes planteada, se puede decir que el desempeño docente es el grado en que el docente dirige sus esfuerzos sobre metas y propósitos concretos, cumpliendo a cabalidad con los requisitos y deberes inherentes a la función que como educador debe llevar a cabo.

Teniendo en cuenta que el desempeño docente es dinámico, que se requiere de ciertas acciones humanas para alcanzar un resultado, se tomaron para su evaluación ciertos factores que se manifiestan a través de su conducta, pudiendo ser observados y medidos en función de su puesto de trabajo. (Ver gráfico 4). En el gráfico 5 se pueden observar los elementos que se tomaron en cuenta en la investigación y que permitieron establecer la relación entre las variables.

\section{La relación, misión y desempeño docente en la Escuela objeto de estudio}

Se planteó una hipótesis general con su respectiva hipótesis nula

$H_{1}$ : La misión de la Escuela Técnica Hno. Ildefonso Gutiérrez direcciona en un sentido significativo el desempeño docente.

$H_{0}$ : La misión de la Escuela Técnica Hno. Ildefonso Gutiérrez no direcciona en un sentido significativo el desempeño docente. 


\section{Gráfico 4}

Evaluación del desempeño docente

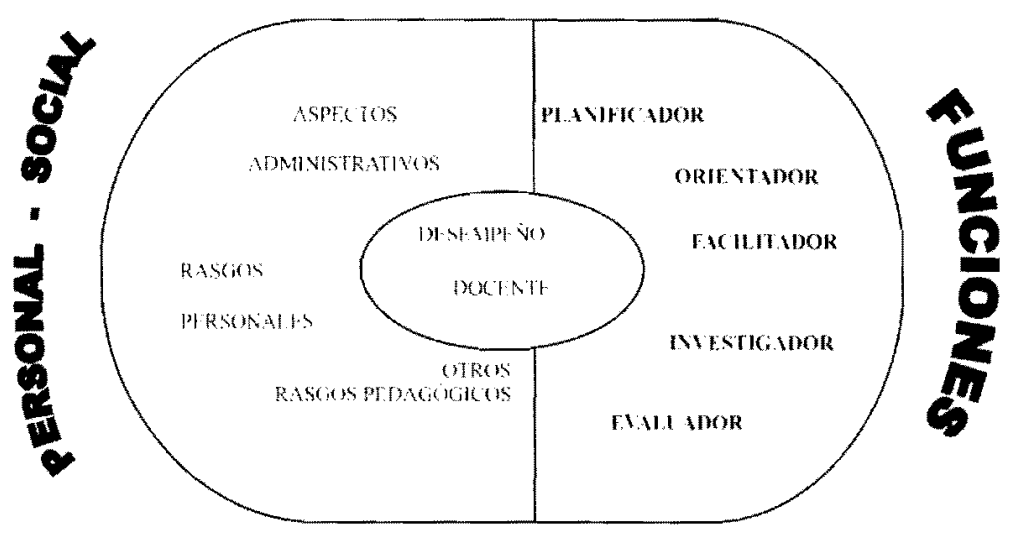

Fuente: Elaboración propia.

Asimismo se formularon dos hipótesis especificas con sus respectivas hipótesis nulas para relacionar el desempeño docente con cada una de las dimensiones que sustentan a la misión (Conocimiento y Valores).

$\mathbf{H}_{2}$ : Existe relación entre el nivel de conocimiento de la misión organizacional con el desempeño de las funciones del docente.

$H_{0}$ : No existe relación entre el conocimiento de la misión organizacional con el desempeño de las funciones del docente.

$\mathrm{H}_{3}$ : Existe relación entre la identificación de los valores corporativos de la institución por parte de los docentes y el desempeño de su labor.

$\mathrm{H}_{0}$ : No existe relación entre la identificación de los valores corporativos de la institución por parte de los docentes y el desempeño de su labor.
Para recolectar la información de la investigación objeto de estudio se aplicó un instrumento mixto a todos los docentes de la institución seleccionada, con la finalidad de medir el nivel de conocimiento que ellos tenian de la misión de la organización e identificaran los valores corporativos. El instrumento poseía en una $1^{\text {era }}$ parte un test de aprovechamiento con ítems tipo múltiple con respuestas óptimas, las cuales de acuerdo a Gronlund (1978:449), constan de un conjunto de ítems en donde las opciones presentadas son parcialmente correctas, pero una es claramente mejor que las demás.

Por otra parte, debido a que uno de los elementos que contiene la misión son los valores corporativos y éstos están muy bien definidos dentro de la organización objeto de estudio y además tiene un gran peso dentro de la misma, se aplicó una $2^{\text {da }}$ parte del instrumento, empleán- 


\section{Gráfico 5}

Elementos: visión, misión, valores corporativos y desempeño docente

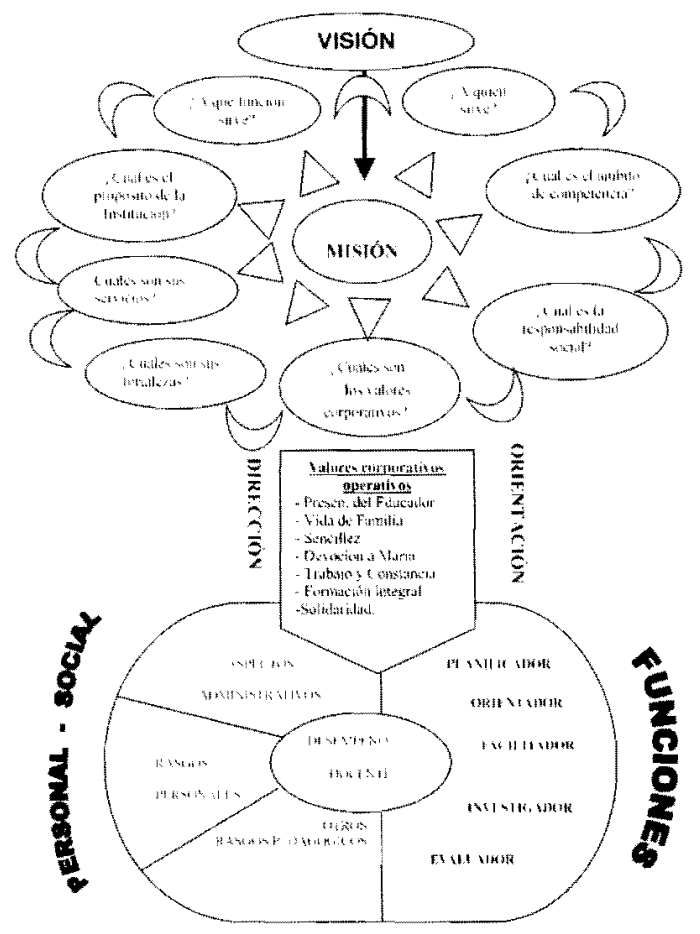

Fuente: Elaboración propia.

dose para ello una escala de fraseo tipo Likert, con la finalidad de que los docentes identificaran con que frecuencia esos valores corporativos se daban dentro de la institución.

De igual forma se diseñó un cuestionario con escalas de acuerdos tipo Likert el cual fue aplicado a 20 alumnos, todos ellos estudiantes del $2^{\text {do }}$ Año del Ciclo Profesional de las Menciones Promoción y Ventas, Dibujo Técnico y Contabilidad de la institución seleccionada, con la finalidad de medir el desempeño de los docentes que laboran en la misma. Esta población fue seleccionada al azar y se seleccionó de las diferentes menciones que egresan de la institución, ya que en el transcurso de $7^{\text {mo }}$ año Básico a $2^{\text {do }}$ año del Ciclo Profesional tienen la oportunidad de recibir clases por parte de todos los docentes que alli laboran.

En correspondencia con el tipo de investigación se aplicó un tratamiento de tipo descriptivo para luego relacionar las variables objeto de estudio. Se procesaron los datos utilizando tablas de doble entrada que permitieron calcular el puntaje total tanto del conocimiento de la misión, valores corporativos y desempeño docente por cada indicador, asi como el 
porcentaje de cada uno de ellos. Acumulándose por un lado la frecuencia de respuestas de cada indicador y por cada sujeto, para luego calcular los respectivos porcentajes. El análisis permitió ubicar las dimensiones y variables en los siguientes niveles porcentuales: $(0 \%-25 \%$ bajo), (26\%-50\% medio), (51\%-75\% alto) y $(76 \%-100 \%$ óptimo).

El análisis de los datos se orientó a darle respuesta a la hipótesis formulada y atender a las dos variables que se estudiaron: Dirección de la Misión Organizacional y Desempeño Docente.

En el gráfico 6 puede apreciarse el porcentaje total de cada uno de los indicadores y el promedio de las dimensiones: conocimiento $(76.79 \%)$ y valores corporativos (82.98\%). Estos resultados permitieron ubicar la variable Dirección de la Misión Organizacional en un nivel óptimo reflejado con un $80.03 \%$, lo que significa que los docentes de la Institución conocen claramente la misión de ésta e identifican en un nivel óptimo los valores corporativos, lo que demuestra que la misión direcciona el desempeño de los miembros de la organización en un nivel óptimo.

En el gráfico 7 se puede apreciar la sumatoria del promedio porcentual de ambas dimensiones Área Personal Social $(91.13 \%)$ y Área de Funciones $(90.68 \%)$, lo que permitió ubicar la variable desempeño docente en un nivel óptimo reflejado con un $90.91 \%$, significando con este indice que los docentes de la Institución objeto de estudio, alcanzan el mayor rango posible en el cumplimiento de sus tareas y funciones.

En la Tabla 1, se puede observar la correlación hecha a cada dimensión (co- nocimiento - valores corporativos) con la variable desempeño docente. Se observa que el coeficiente de correlación en cuanto a la dimensión conocimiento es $r=0.59$, para un nivel de probabilidad de error $p$ 0.001 , indicando con esto que existe una relación muy significativa entre el conocimiento de la misión organizacional y el desempeño docente, lo que demuestra que de cada 1.000 casos donde se establezca la relación entre (Conocimiento y Desempeño Docente) ésta se da en 999 sujetos y en sólo 1 caso la asociación se da al azar. Esta relación expresada a través de un nivel de confianza de $99.96 \%$, indica que se acepta la hipótesis $\mathrm{H}_{2}$, lo que evidencia que a mayor nivel de conocimiento de la misión de la institución por parte de los docentes hay un nivel mayor de desempeño en sus funciones.

En cuanto a los valores corporativos puede corroborarse que el coeficiente de correlación en cuanto a esta dimensión es $r=0.36$, no alcanza el coeficiente de correlación de la dimensión anterior, más sin embargo es significativo a un nivel de $\mathrm{p} 0.05$, evidenciándose que existe una relación estadísticamente significativa entre los valores corporativos de la institución y el desempeño docente, lo que demuestra que de cada 100 casos donde se estudie la relación entre (Valores Corporativos y Desempeño Docente) esta asociación se evidencia en 99 sujetos y en un solo caso se da al azar. Esta relación es expresada a través de un nivel de confianza de $98.31 \%$, lo que evidencia que se acepta la hipótesis $\mathrm{H}_{3}$, indicando con esto que a mayor nivel de identificación de los valores corporativos de la Institución por parte de los docentes, hay un desempeño mejor en su labor. 


\section{Grático 6}

Distribución porcentual de la variable dirección de la misión organizacional de la Escuela Técnica Hno. Ildefonso Gutiérrez

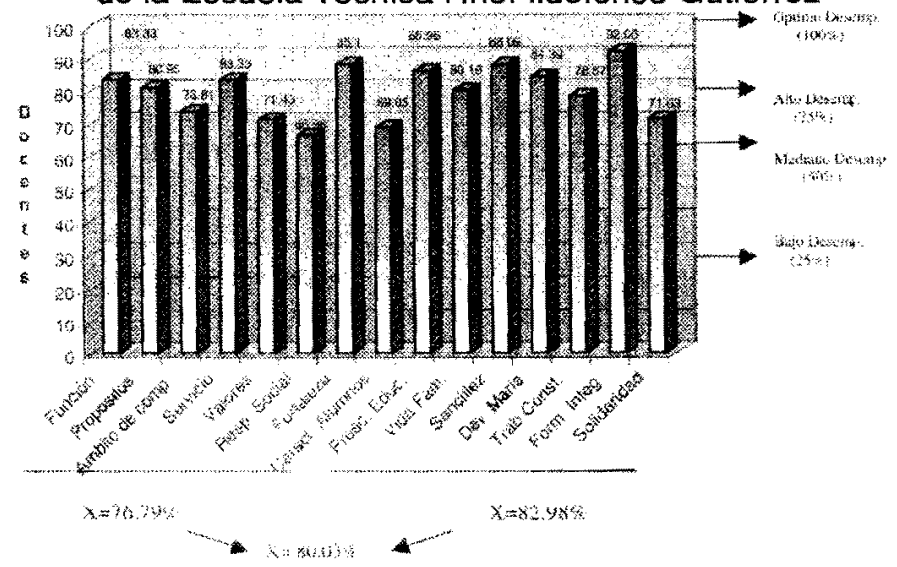

\section{Gráfico 7}

Distribución porcentual de la variable desempeño docente

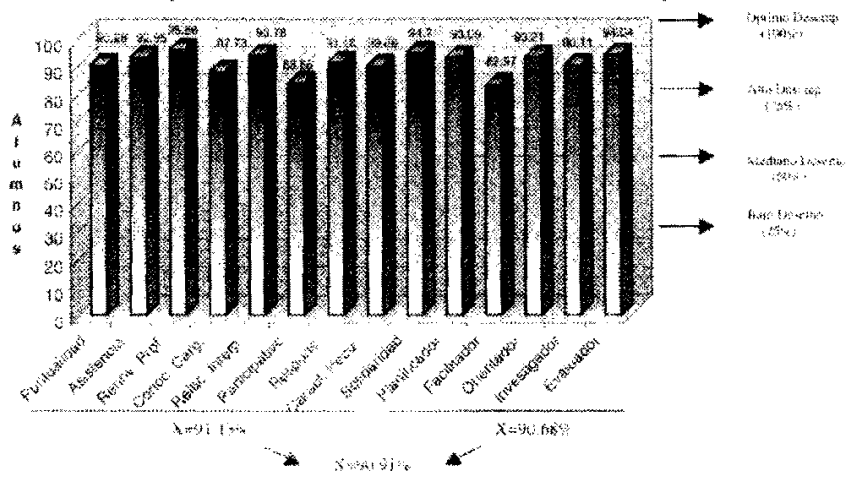

Tabla 1

Correlación de las variables (misión y desempeño docente)

\begin{tabular}{lccc}
\multicolumn{1}{c}{ Par de variables } & Valid. & $\begin{array}{c}\text { Coeficiente de } \\
\text { Spearmean }\end{array}$ & $\begin{array}{c}\text { Nivel de } \\
\text { Probabilidad }\end{array}$ \\
\hline $\begin{array}{l}\text { Desempeño } \\
\text { y conocimiento }\end{array}$ & 42 & $\mathrm{~A}$ & \\
$\begin{array}{l}\text { Desempeño y valores } \\
\text { corporativos }\end{array}$ & 42 & 0.590760 & 0.00038 \\
Desempeño y misión & 42 & 0.367014 & 0.016810 \\
\hline
\end{tabular}


De igual manera se puede observar el coeficiente de correlación entre las dos variables de la investigación (misión y desempeño docente), el cual es $r=0.43$, para un nivel de probabilidad de $\mathrm{p} 0.001$, indicando con esto que existe una relación muy signíficativa entre la misión de la organización objeto de estudio y el desempeño de sus docentes, lo que demuestra que de cada 1.000 casos donde se establezca la relación (Dirección de la Misión y el Desempeño Docente) ésta se da en 999 casos y en sólo 1 se da al azar. Esta relación es expresada con un nivel de confianza de $99.61 \%$, por lo que se acepta la hipótesis general en donde se establece que la misión de la Escuela Técnica Hno. Ildefonso Gutiérrez, direcciona en un nivel estadisticamente significativo el desempeño docente.

\section{Conclusiones}

Se determinó que la Misión Organizacional de la Escuela Técnica Hno. Ildefonso Gutiérrez direcciona el desempeño de los docentes que alli laboran, reafirmándose la teoría de Davis (1994), Quigley (1996), Albrecht (1996) Serna Gómez (1997), que la misión es la formulación de los propósitos de una organización que la distingue de otras de acuerdo con sus valores, servicios, ámbito de competencia, responsabilidad social, fortalezas. Fueron estos elementos de la misión los que sirvieron de marco de relerencia para demostrar que en la medida que los docentes conocen la misión de la Institución, hay mayor nivel de desempeño docente, evidenciándose con esto que existe un alto com- promiso por parte de sus miembros para cumplir con las tareas y funciones asignadas por la organización.

Se comprobó que el conocimiento de la misión por parte de los docentes es fundamental para el desempeño de sus funciones. De igual forma se pudo observar que los docentes que tienen menos tiempo en la Institución tienen un nivel de conocimiento más bajo que los docentes que tienen más años de servicios, por lo que se asume que el conocimiento y la internalización de la misión no se adquiere en poco tiempo, por lo que se hace necesaria una buena selección del personal que ingrese a la organización, de tal manera que su perfil de competencia y valores individuales se correspondan con los esperados dentro de la organización, logrando así que la identificación y el conocimiento en cuanto a la misión sea más rápido y efectivo.

Esta observación, adquiere cierta relevancia si se toma en consideración que el recurso humano y su desempeño es lo más importante en una organización, ya que de éste depende el logro de sus propósitos y objetivos. Por lo que se hace necesario insistir en la misión, como uno de los elementos que impacta y direcciona el desempeño docente. Este aspecto plantea el por qué no se debe formar, ni direccionar los recursos humanos en las instituciones educativas desvinculados del conocimiento de sus propósitos, función y servicio de las organizaciones.

Se determinó que los valores constituyen un elemento importante en la dirección de la misión, ya que a medida que los docentes se identifican con los valores corporativos adquieren un alto grado 
de compromiso de cumplir con su labor, por lo que se asume que los valores constituyen la guía y dirección para lograr la misión por un camino más corto y exitoso.

Toda organización ha de tener definido dos grandes grupos de valores o principios compartidos que orientan sus objetivos de acción cotidiana; tal es el caso de los valores finales asociados a su visión y misión y los valores operativos o instrumentales, asociados con las acciones del quehacer diario, manera de pensar y hacer las cosas en la organización, lo que constituyen los valores tácticos que reguIan la conducta cotidiana de los miembros de la Institución para alcanzar la misión y visión de la misma.

En la Escuela Técnica Hno. Ildefonso Gutiérrez se evidencia esta teoría, ya que la misión ha sido direccionada por los valores, pues el fundador Marcelino Champagnat con un sentido visionario, adelantado a su tiempo, empleó este sistema de valores que hoy es corroborado por las nuevas teorías gerenciales.

De igual forma se reafirmó lo expuesto por Robbins (1996), cuando manifiesta que existe una relación positiva y significativa entre el desempeño y la manera como los individuos perciben el ambiente de trabajo, lo que expresa que uno de los factores que contribuye a mejorar el desempeño es el hecho que los valores del empleado armonicen con los valores de la empresa. Este aspecto fue corroborado en esta investigación, ya que los docentes de la Escuela Técnica Hno. Ildefonso Gutiérrez identifican y comparten casi siempre los valores corporativos de la misma, conocen la misión y se sienten motivados a cumplir con su labor por lo que se desempeñan en un nivel óptimo cumpliendo alto grado con su función; sin que ésta sea exigencia de la organización.

\section{Bibliografía citada}

Albrecht, Karl (1996). La Misión de la Empresa. Ediciones Piados lbérica. Buenos Aires.

Dalziel, Murray M., Cubeiro, Juan Carlos y Fernández, Guadalupe (Coordinadores) (1996). Las Competencias: Clave para una Gestión Integrada de los Recursos Humanos. Ediciones Deusto, S.A. Bilbao, España.

David, Fred (1994). La Gerencia Estratégica. Fondo Editorial Legis. U.S.A

Escorihuela, Josep $M^{a}$ et al (1983). El Educador Marista. Editonal Luis Vives. España.

Garcia, Salvador y Dolán, Shimon (1997). La Dirección por Valores. Editorial McGraw-Hill. Madrid, España.

Gronlund, Lund Norman (1978). Elaboración de Tests de Aprovechamiento, Editorial Trillas. México.

Congreso de la República (1980). Ley Orgánica de Educación. Gaceta Oficial No 2635 .

Milkovichg (1994). Dirección y Administración de los Recursos Humanos. USA. Edit. Addison. Wesley. Iberoamericana. México.

Presidencia de la República. Reglamento de la Prolesión Docente (1991). Gaceta Oficial de la República de Venezuela No. 4338. Eduven. Caracas, Venezuela.

Quigley, Joseph (1996). Visión. D'Vinni Editorial Colombia

Robbins, Stephen (1996). Comportamiento Organizacional. Séptima Edición. Prentice-Hall, Hispanoamericana. México.

Serna G., Humberto (1997). Planeación y Gestión Estratégica. Ram Editores. Santafé de Bogotá, Colombia. 